\title{
Vertical Vibration Control of Cold Rolling Mill's Rolls Based on Time-delay Feedback Control Method
}

\author{
Dongxiao Hou \\ School of Control Engineering, Northeastern University at Qinhuangdao, China
}

\begin{abstract}
In this paper, a two degree of freedom nonlinear vertical vibration equation of the cold rolling mill with the dynamic rolling force was established, then the delay feedback control method was introduced into the equation to controlled the vertical vibration of the system. The amplitude-frequency equations of primary resonance of system was carried out by using the multi-scale method, and the resonance characteristics of different parameters of delay feedback control method were obtained by adopting the actual parameters of rolling mill. It is found that the size of the resonance amplitude value was effectively controlled and the resonance region and jumping phenomenon of the system were eliminated by selecting the appropriate time-delay parameters combination, which provides an effective theoretical reference for solving mill vibration problems.
\end{abstract}

\section{Introduction}

The vibration of rolling mill often occurs in rolling process. The occurrence of vibration not only affects the quality of rolling products, but also leads to breakdown of the rolling equipment. There are mainly two kinds of vibration in rolling mill, namely torsional vibration of main drive system and vertical vibration of rolls. Especially, the vertical vibration of rolls directly effects the exit thickness of strip. In order to restrain vertical vibration of rolls and improve stability of rolling process, the active control theory and technology has been used and to control the vertical vibration phenomenon. Zhang adopted the robust theory to control the strip tension of cold rolling mill and then inhibited the occurrence of vertical vibration [1]. Fuzzy neural network is used in hydraulic AGC system and the accuracy of strip is improved[2]. Time-delay phenomenon is very common in the control system. Plenty of researches have been studied on it in different research fields [3, 4]. Timedelay phenomenon also exists in the control system of rolling mill.

In this paper, in order to control resonance characteristics of rolling mill, the time-delay feedback control method is applied to the two degrees of freedom vertical vibration equation of cold rolling mill. By using the multi-scale method, the primary resonance amplitudefrequency characteristic equation is solved out, and the impact of time-delay parameters on the primary resonance are studied. The simulation results indicate that the size of resonance amplitude is effectively controlled, and the resonance region and jumping phenomenon of the system are eliminated.

\section{The time-delay feedback control model of rolling mill's two degree freedom vibration system}

Considering the effects of dynamic rolling force [5] and the up-down symmetry of rolling mills, a two degree of freedom nonlinear vertical vibration model of rolling mill is established in Figure 1.

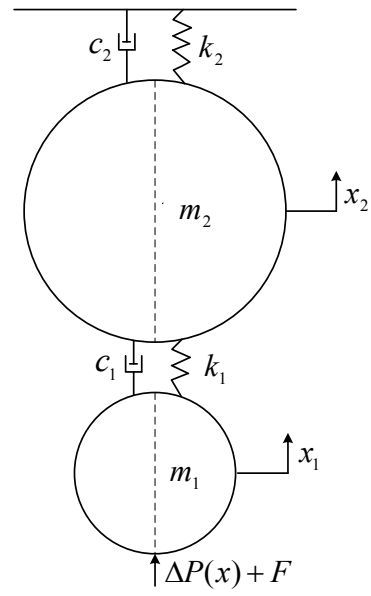

Figure 1. The mechanical vertical vibration model of rolls with nonlinear dynamic force.

The nonlinear dynamic equation of rolling mill in Figure 1 can be established as

$$
\left\{\begin{array}{l}
m_{1} \ddot{x}_{1}+c_{1}\left(\dot{x}_{1}-\dot{x}_{2}\right)+k_{1}\left(x_{1}-x_{2}\right)=\Delta P(x)+F \\
m_{1} \ddot{x}_{2}+k_{2} x_{2}+c_{2} x_{2}-k_{1}\left(x_{1}-x_{2}\right)-c_{1}\left(\dot{x}_{1}-\dot{x}_{2}\right)=0
\end{array}\right.
$$


Where, $m_{1}$ and $m_{2}$ are defined as the equivalent mass of top work roll and top back-up roll, respectively. $k_{1}$ is defined as equivalent stiffness between the top work roll and top back-up roll. $k_{2}$ and $c_{2}$ are defined as equivalent stiffness and equivalent damping between the top back-up roll and top beam of mill housing, respectively. $F$ is defined as external excitation. $\Delta P(x)$ is dynamic variation of rolling force, $\Delta P(x)=b_{1} x+b_{2} x^{2}+b_{3} x^{3}, x=2 x_{1}$.

The time-delay feedback control is applied in equation (1) and it can be expressed as

$$
\left\{\begin{array}{c}
m_{1} \ddot{x}_{1}+c_{1}\left(\dot{x}_{1}-\dot{x}_{2}\right)+k_{1}\left(x_{1}-x_{2}\right)=\Delta P(x)+F \\
\quad+g_{1} x_{1}\left(t-\tau_{1}\right)+g_{2} \dot{x}_{1}\left(t-\tau_{2}\right) \\
m_{1} \ddot{x}_{2}+k_{2} x_{2}+c_{2} \dot{x}_{2}-k_{1}\left(x_{1}-x_{2}\right)-c_{1}\left(\dot{x}_{1}-\dot{x}_{2}\right)=0
\end{array}\right.
$$

Where, $\tau_{1}$ and $\tau_{2}$ are time-delay parameters, $g_{1}$ and $g_{2}$ are gain factor.

The equation (2) can be simplified as

$$
\left\{\begin{array}{l}
\ddot{x}_{1}+\omega_{1}^{2} x_{1}=\alpha_{1} x_{2}+\beta_{1}\left(\dot{x}_{1}-\dot{x}_{2}\right)+\gamma_{1} x_{1}^{2} \\
\quad+\eta_{1} x_{1}^{3}+F_{0}+d_{1} x_{1}\left(t-\tau_{1}\right)+d_{2} \dot{x}_{1}\left(t-\tau_{2}\right) \\
\ddot{x}_{2}+\omega_{2}^{2} x_{2}=\alpha_{2} x_{1}+\beta_{2} \dot{x}_{1}+\gamma_{2} \dot{x}_{2}
\end{array}\right.
$$

Where, $\omega_{1}^{2}=\left(k_{1}-2 b_{1}\right) / m_{1}, \quad \omega_{2}^{2}=\left(k_{1}+k_{2}\right) / m_{2}$, $\alpha_{1}=k_{1} / m_{1}, \beta_{1}=-c_{1} / m_{1}, \gamma_{1}=4 b_{2} / m_{1}, \eta_{1}=8 b_{3} / m_{1}$, $\alpha_{2}=k_{1} / m_{2} \quad, \quad \beta_{2}=c_{1} / m_{2} \quad, \quad \gamma_{2}=-\left(c_{1}+c_{2}\right) / m_{2}$, $d_{1}=g_{1} / m_{1}, \quad d_{2}=g_{2} / m_{1}, F_{0}=F / m_{1}$.

\section{Primary resonance analysis}

Assuming equation (3) is a small nonlinear system, the right side of the equation (3) multiply by small parameter

$$
\left\{\begin{array}{c}
\ddot{x}_{1}+\omega_{1}^{2} x_{1}=\varepsilon\left[\alpha_{1} x_{2}+\beta_{1}\left(\dot{x}_{1}-\dot{x}_{2}\right)+\gamma_{1} x_{1}^{2}\right. \\
\left.\quad+\eta_{1} x_{1}^{3}+F_{0}+d_{1} x_{1}\left(t-\tau_{1}\right)+d_{2} \dot{x}_{1}\left(t-\tau_{2}\right)\right] \\
\ddot{x}_{2}+\omega_{2}^{2} x_{2}=\varepsilon\left[\alpha_{2} x_{1}+\beta_{2} \dot{x}_{1}+\gamma_{2} \dot{x}_{2}\right]
\end{array}\right.
$$

In the case of primary resonance, $F_{0}=F_{1} \cos (\omega t)$, set $\omega_{1}^{2}=\omega^{2}+\varepsilon \sigma$, and substituting it in equation (4)

$$
\left\{\begin{array}{c}
\ddot{x}_{1}+\omega^{2} x_{1}=\varepsilon\left[\alpha_{1} x_{2}+\beta_{1}\left(\dot{x}_{1}-\dot{x}_{2}\right)+\gamma_{1} x_{1}^{2}+\eta_{1} x_{1}^{3}\right. \\
\left.\quad+F_{0}+d_{1} x_{1}\left(t-\tau_{1}\right)+d_{2} \dot{x}_{1}\left(t-\tau_{2}\right)-\sigma x_{1}\right] \\
\ddot{x}_{2}+\omega_{2}^{2} x_{2}=\varepsilon\left[\alpha_{2} x_{1}+\beta_{2} \dot{x}_{1}+\gamma_{2} \dot{x}_{2}\right]
\end{array}\right.
$$

Where, $\sigma$ is frequency modulation parameter,

By using multiple scales method, assuming it has the first-order approximate solution as follows

$$
\left\{\begin{array}{l}
x_{1}=x_{11}\left(T_{0}, T_{1}\right)+\varepsilon x_{12}\left(T_{0}, T_{1}\right) \\
x_{2}=x_{21}\left(T_{0}, T_{1}\right)+\varepsilon x_{22}\left(T_{0}, T_{1}\right)
\end{array}\right.
$$

Substituting equation (6) into equation (5), separating terms each order of $\varepsilon$, it has

$$
\left\{\begin{array}{l}
D_{0}^{2} x_{11}+\omega^{2} x_{11}=0 \\
D_{0}^{2} x_{21}+\omega_{2}^{2} x_{21}=0
\end{array}\right.
$$

$$
\left\{\begin{array}{l}
D_{0}^{2} x_{12}+\omega^{2} x_{12}=-2 D_{0} D_{1} x_{11}+\alpha_{1} x_{21}+\beta_{1} D_{0}\left(x_{11}-x_{21}\right) \\
\quad+\gamma_{1} x_{11}^{2}+\eta_{1} x_{11}^{3}+F_{1} \cos \omega t+d_{1} x_{11}\left(t-\tau_{1}\right) \\
\quad+d_{1} D_{0} x_{11}\left(t-\tau_{2}\right)-\sigma x_{11} \\
D_{0}^{2} x_{22}+\omega_{2}^{2} x_{22}=-2 D_{0} D_{1} x_{21}+\alpha_{2} x_{11}+\beta_{2} D_{0} x_{11}+\gamma_{2} D_{0} x_{21}
\end{array}\right.
$$

Set the solution of equation (7) was

$$
\left\{\begin{array}{l}
x_{11}=A_{1}\left(T_{1}\right) e^{i \omega T_{0}}+\varepsilon \bar{A}_{1}\left(T_{1}\right) e^{-i \omega T_{0}}+c c \\
x_{21}=A_{2}\left(T_{1}\right) e^{i \omega_{2} T_{0}}+\varepsilon \bar{A}_{2}\left(T_{1}\right) e^{-i \omega_{2} T_{0}}+c c
\end{array}\right.
$$

Substituting equation (8) into equation (7), the following equation can be expressed as

$$
\left\{\begin{array}{l}
D_{0}^{2} x_{12}+\omega^{2} x_{12}=\left(-2 i \omega D_{1} A_{1}+i \omega \beta_{1} A_{1}+3 \eta_{1} A_{1}^{2} \bar{A}_{1}+0.5 F_{1}\right. \\
\left.\quad+d_{1} A_{1} e^{-i \omega \tau_{1}}+i \omega d_{2} A_{1} e^{-i \omega \tau_{2}}-\sigma A_{1}\right) e^{i \omega T_{0}}+\left(\alpha_{1} A_{2}\right. \\
\left.\quad-i \omega_{2} \beta_{1} A_{2}\right) e^{i \omega_{2} T_{0}}+\gamma_{1} A_{1}^{2} e^{i 2 \omega T_{0}}+\eta_{1} A_{1}^{3} e^{i 3 \omega T_{0}}+2 \gamma_{1} A_{1} \bar{A}_{1}+c c \\
D_{0}^{2} x_{22}+\omega_{2}^{2} x_{22}=\left(-2 i \omega_{2} D_{1} A_{2}+i \omega_{2} \gamma_{2} A_{2}\right) e^{i \omega_{2} T_{0}} \\
\quad+\left(\alpha_{2} A_{1}+i \omega \beta_{2} A_{1}\right) e^{i \omega T_{0}}+c c
\end{array}\right.
$$

The secular term of equation (10) can be obtained as

$$
\left\{\begin{array}{l}
-2 i \omega D_{1} A_{1}+i \omega \beta_{1} A_{1}+3 \eta_{1} A_{1}^{2} \bar{A}_{1}+0.5 F_{1} \\
+d_{1} A_{1} e^{-i \omega \tau_{1}}+i \omega d_{2} A_{1} e^{-i \omega \tau_{2}}-\sigma A_{1}=0 \\
-2 i \omega_{2} D_{1} A_{2}+i \omega_{2} \gamma_{2} A_{2}=0
\end{array}\right.
$$

The polar coordinate of $A_{1}$ and $A_{2}$ of the equation (11) is introduced as follows

$$
\begin{aligned}
& A_{1}=0.5 a\left(T_{1}\right) e^{i \varphi_{1}\left(T_{1}\right)} \\
& A_{2}=0.5 b\left(T_{1}\right) e^{i \varphi_{2}\left(T_{1}\right)}
\end{aligned}
$$

Substituting equation (12) into equation (11), separate the real part and the imaginary part and get

$$
\left\{\begin{aligned}
\dot{a}= & \frac{\varepsilon}{2 \omega}\left[a \beta_{1} \omega-F_{1} \sin \varphi_{1}-d_{1} a \sin \left(\omega \tau_{1}\right)\right. \\
& \left.\quad+a \omega d_{2} \cos \left(\omega \tau_{2}\right)\right] \\
\dot{\varphi}_{1}= & -\frac{\varepsilon}{2 a \omega}\left[\frac{3}{4} \eta_{1} a^{3}+F_{1} \cos \varphi_{1}+d_{1} a \cos \left(\omega \tau_{1}\right)\right. \\
& \left.\quad+a \omega d_{2} \sin \left(\omega \tau_{2}\right)-a \sigma\right] \\
\dot{b}= & \frac{1}{2} \varepsilon \gamma_{2} b \\
\dot{\varphi}_{2}= & 0
\end{aligned}\right.
$$

In the steady-state, existing $\dot{a}=\dot{b}=\dot{\varphi}_{1}=\dot{\varphi}_{2}=0$, eliminate $\varphi_{1}$ and $\varphi_{2}$, then the primary resonance amplitude-frequency equation of the controlled equation of system can be obtained as

$$
\begin{aligned}
& {\left[\frac{3}{4} \eta_{1} a^{3}+d_{1} a \cos \left(\omega \tau_{1}\right)+a \omega d_{2} \sin \left(\omega \tau_{2}\right)-a \frac{\omega_{1}^{2}-\omega^{2}}{\varepsilon}\right]^{2}} \\
& +\left[a \beta_{1} \omega-d_{1} a \sin \left(\omega \tau_{1}\right)+a \omega d_{2} \cos \left(\omega \tau_{2}\right)\right]^{2}=F_{1}^{2}
\end{aligned}
$$




\section{Simulation}

Taking the actual structure parameters and technological parameters of the rolling mill as an example, the detail parameters are as follows:

$m_{1}=2.182 \times 10^{4} \mathrm{~kg}, \quad m_{2}=1.225 \times 10^{5} \mathrm{~kg}, \quad k_{1}=6.0186 \times 10^{10}$ $\mathrm{N} / \mathrm{m}, \quad k_{2}=3.1825 \times 10^{10} \mathrm{~N} / \mathrm{m}, \quad c_{1}=1.01 \times 10^{6} \quad \mathrm{~N} \cdot \mathrm{s} / \mathrm{m}$, $c_{2}=1.25 \times 10^{6} \mathrm{~N} \cdot \mathrm{s} / \mathrm{m}, F_{0}=0.5 \times 10^{6} \mathrm{~N}$.

The nonlinear parameters of dynamic rolling force are as follows:

$b_{1}=-4.478 \times 10^{9} \mathrm{~N} / \mathrm{m}, \quad b_{2}=-2.910 \times 10^{12} \mathrm{~N} / \mathrm{m}^{2}, \quad b_{3}=-$ $3.256 \times 10^{15} \mathrm{~N} / \mathrm{m}^{3}, \varepsilon=0.01$.

Figures 2 illustrates primary resonance amplitudefrequency curves in different delay-time parameters.

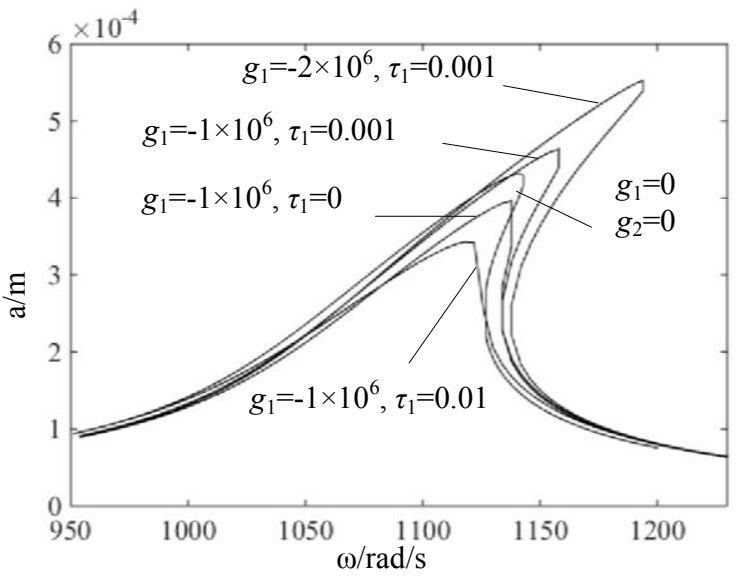

(a) $g_{2}=-1 \times 10^{3}$ and $\tau_{2}=0.001$

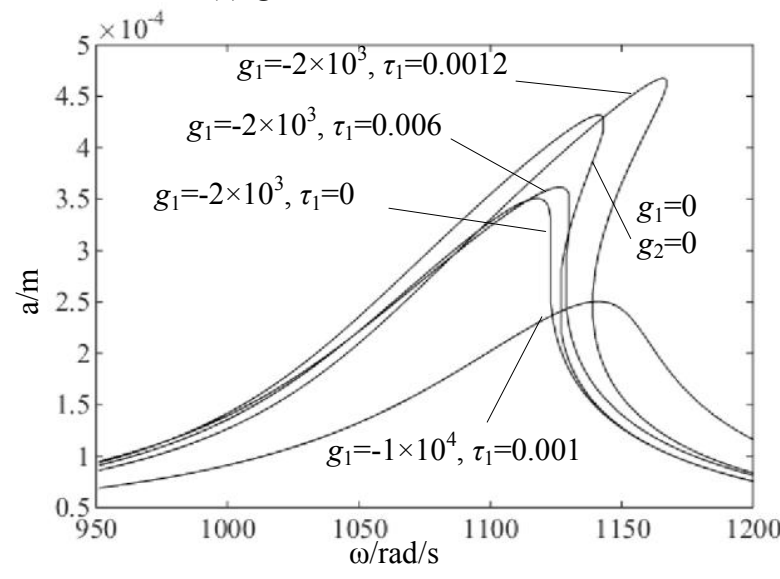

(b) $g_{1}=-1 \times 10^{6}$ and $\tau_{1}=0.001$

Figure 2. Primary resonance amplitude-frequency curves.

Figure 2(a) is the primary resonance amplitudefrequency curves of system when $g_{1}$ and $\tau_{1}$ take different combinations and the time delay parameters $g_{2}=-1 \times 10^{3}$, $\tau_{2}=0.001$. When $g_{1}=0$ and $g_{2}=0$, it is the primary resonance amplitude-frequency curve of original system. If $\tau_{1}=0$ (namely no delay), when $g_{1}=-1 \times 10^{6}$, the amplitude and resonance zone decreases. As $\tau_{1}$ increased, the amplitude and resonance zone increases firstly, then the amplitude and resonance zone decreases, it means adjusting the parameters $g_{1}$ and $\tau_{1}$ can effectively control the size of the resonance amplitude values.

Figure 2(b) illustrates the primary resonance amplitude -frequency curves of system when $g_{2}$ and $\tau_{2}$ take different combinations and the time delay parameters $g_{1}=-1 \times 10^{6}$, $\tau_{1}=0.001$. when $g_{2}$ is constant and $\tau_{2}$ increases, firstly the amplitude decreases with $\tau_{2}$ is no delay, then the amplitude increases with $\tau_{2}$ increases. When $\tau_{2}$ increase to a certain value, the amplitude decreases. when $g_{2}$ increases, the amplitude and resonance zone decreases.

Figures 3 are the time-domain curves of $m_{1}$ in the case of no control and control condition with $\omega$ is equal to $1140 \mathrm{rad} / \mathrm{s}$.

Figure 3(a) is $g_{1}$ and $g_{2}$ are both equal to zero, namely no control. Figure3 (b) is time-domain curve in control, it can be seen that the resonance amplitude is less than no control, it means it could be improve resonance problem by adopting time-delay feedback method. So it can be seen adjusting the time-delay parameters can effectively reduce the amplitude of system, eliminate the resonance phenomenon and make the system tend to be stable more quickly.

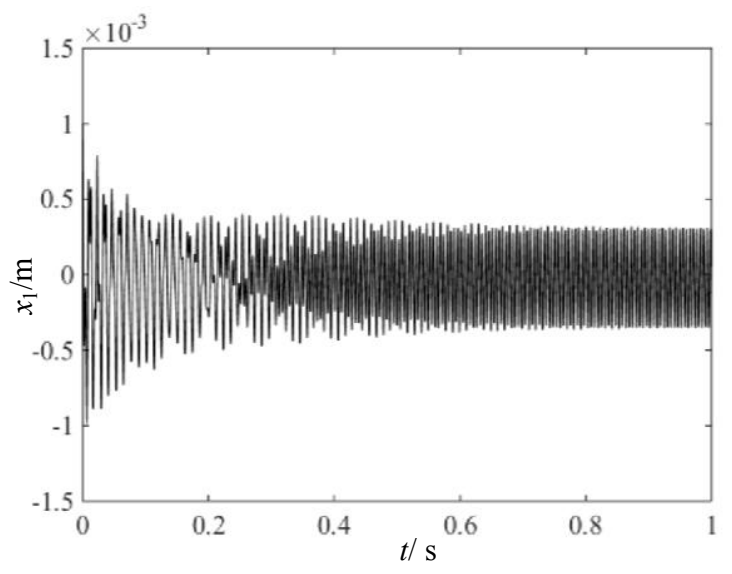

(a) $g_{1}=0, g_{2}=0, \tau_{1}=0, \tau_{1}=0$

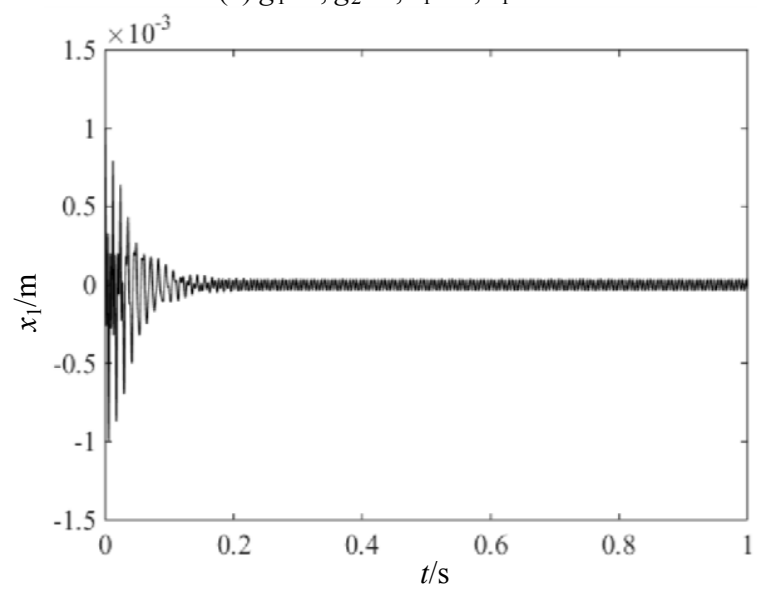

(b) $g_{1}=-2 \times 10^{6}, g_{2}=-1 \times 10^{3}, \tau_{1}=0.001, \tau_{1}=0.001$

Figure 3. The time-domain curves of $m_{1}$ with uncontrolled and controlled.

In summary, by using the time-delay feedback method to control the two degree of freedom vibration system and selecting the appropriate time-delay feedback parameters can effectively improve the primary resonance amplitude frequency characteristic of system and make the mill system in more stable state. 


\section{Conclusion}

This paper firstly established a two degree of freedom vibration equation of the cold rolling mill with the dynamic rolling force. then the delay feedback control method is introduced into the equation to control the vertical vibration of system. In addition, the primary resonance amplitude-frequency characteristic controlled equation of system is solved out and the effect of timedelay parameters on the primary and internal resonance are analyzed.

The appropriate time-delay parameter combination is selected to effectively control the amplitude value of resonance. It eliminates the internal resonance region and jumping phenomenon of system, improved the stability of system, which provides an effective theoretical reference for the study of inhibiting the mill vibration problems.

\section{Acknowledgments}

This research is supported by National Natural Science Foundation of China (Grant No. 51405068), Natural Science Foundation of Hebei Province of China (Grant No.E2014501006), and Technology Research Project of Colleges and Universities of Hebei Province of China (Grant No. 2014202).

\section{References}

1. Zhang X.F Robust Control of Strip Tension for Tandem Cold Rolling Mill. Mater Thesis, University of Science and Technology Beijing, China (2008)

2. Yang $Y$ and Zhang X.M Fuzzy Neural Networks Control for Hydraulic AGC System of Aluminum Cold Rolling Mill. In: Proceedings of 2008 3rd International Conference on Intelligent System and Knowledge Engineering(ISKE 2008), Xiamen, China, 17-19 November 2008, pp. 92-97. Piscataway: Inst. of Elec. and Elec. Eng. (2008)

3. Liu S, Liu B, Zhang Y.K, et al. Hopf bifurcation and stability of periodic solutions in a nonlinear relative rotation dynamical system with time delay. Acta Physica Sinica 59(1):38-43 (2010)

4. Shi P.M, Li J.Z, Liu B, et al. Stability and timedelayed feedback control of a relative-rotation nonlinear dynamical system under quasic-periodic parametric excitation. Acta Physica Sinica 60(9):094501(2011)

5. Hou D.X, Zhu Y and Liu H.R. Research on Nonlinear Vertical Vibration Characteristics of Cold Rolling Mill Based on Dynamic Rolling Force. Chinese Journal of Mechanical Engineering 49(14):45-50. (2013) 